\title{
Atomic Force Microscopy as a Potential Diagnostic Technique in Staphylococcal Infections
}

\author{
F.M. Coldren, ${ }^{*}$ E. Palavecino, ${ }^{* *}$ D.L. Carroll* \\ * Center for Nanotechnology and Molecular Materials, Department of Physics, Wake Forest \\ University, 100 Olin Physical Laboratory, 7507 Reynolda Station, Winston-Salem, NC 27109-7507 \\ ** Department of Pathology, Wake Forest University Medical School, Medical Center Boulevard, \\ Winston-Salem, NC 27157-1072
}

Staphylococcus aureus causes a variety of minor human diseases, particularly skin infections, but also is responsible for severe invasive infections. S. aureus possesses an arsenal of virulence factors that permits it to persist in human infections, including the formation of polysaccharide-rich extracellular matrices. In $S$. aureus eleven serologically distinct capsular polysaccharides have been demonstrated, varying in composition and structure. The role of extracellular polysaccharide matrices in infection is hypothesized to allow bacteria to stick to surfaces, prevent recognition by the immune system, and filter bactericidal substances [1]. The precise role of such matrices remains an enigma, being one or a combination of hypothesized roles.

The introduction of atomic force microscopy has permitted the investigation of biological systems on the cellular level, rather than on the population level. Diminishing antimicrobial treatment options for staphylococcal infections resulting from a prevalence of antibiotic resistant strains demands an understanding in order to combat this organism. Our goal was to investigate surface interactions and mechanical response of three $S$. aureus strains. ATCC strains 10832, 49230, and 13709 of S. aureus were investigated using the atomic force microscope. ATCC 10832 was selected due to its lack of an extracellular matrix [2], while ATCC 49230 and 13709 were chosen for the presence of an extracellular matrix and pathogenic histories [3-4]. Susceptibility tests on the three strains revealed that all strains were susceptible to the antimicrobial agents used to treat staphylococcal infections.

The AFM investigation consisted of topographical imaging and force curves. The topographical imaging completed in non-contact mode produced high resolution images demonstrating dramatic differences in surface morphology of the three staphylococcal strains, strongly dependent on the quantity of extracellular matrix present. Quantitative mechanical response data was obtained by collecting force curves on the individual cells. The responsiveness of the strains to a force varied as well as the adhesive surface properties of each cell. The results of mechanical characterization of three $S$. aureus strains suggest that atomic force microscopy may be highly sensitive to capsule presence and type, permitting potential development into a diagnostic technique.

\section{References}

[1] J. W. Costerton et al., Ann. Rev. Microbiol. 35 (1981) 299.

[2] T. T. Tollersrud et al., APMIS. 109 (2001) 541.

[3] T. Arizono et al., J. Bac. 173 (1991) 4333.

[4] A. F. Gillaspy et al., Infect. Immun. 63 (1995) 3373.

[5] The authors thank the Department of Orthopaedic Surgery at WFU School of Medicine for helpful discussions 

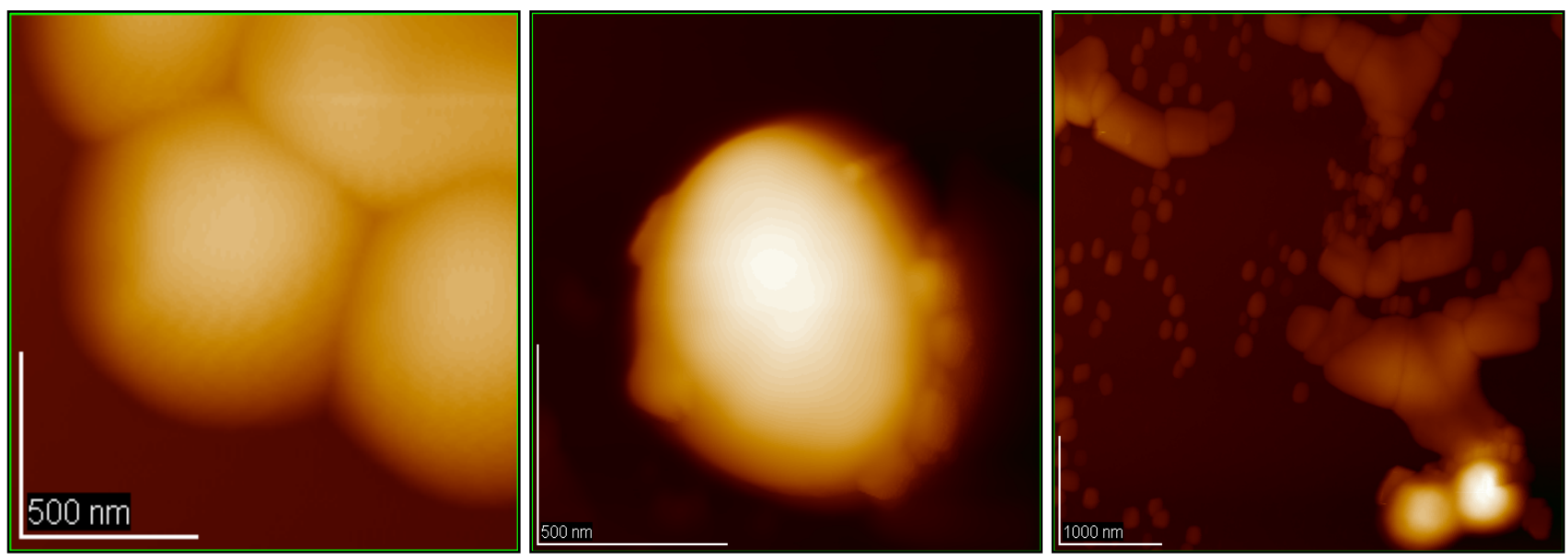

Fig.1. Tapping mode topographical images of ATCC 10832 non-capsulated strain (A), ATCC 49230 capsulated (B), ATCC 13709 capsulated (C). The non-capsulated strain (A) displays smooth topography and distinct borders between cells, while the capsulated strains (B) and (C) have bumpy morphology and indistinct cell borders.

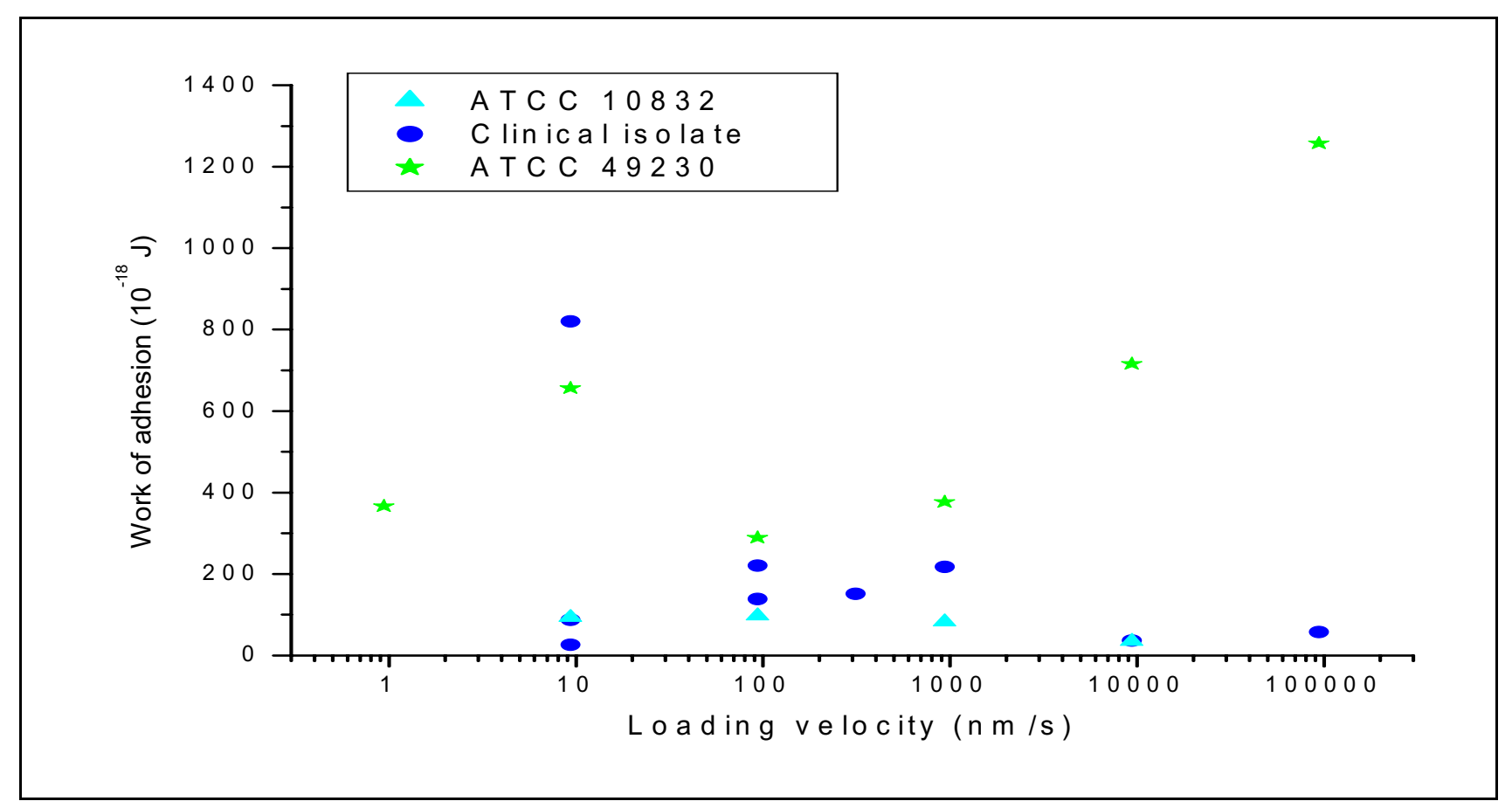

Fig.2 The work of adhesion, the energy required to remove the AFM cantilever from the bacterial surface, was plotted for two of the ATCC strains and a clinical isolate from Wake Forest University School of Medicine. This figure shows that more energy is required to separate the AFM cantilever from ATCC 49230 than the other strains investigated. The energy of interaction is a potential diagnostic tool 